\title{
Perfil epidemiológico de anomalias congênitas no Estado da Bahia
}

\author{
Epidemiological Profile of Congenital Anomalies in the State of Bahia
}

\author{
Aline do Nascimento Andrade ${ }^{1}$, Rita Maria Alves², Maria Betânia Pereira Toralles ${ }^{3 *}$ \\ ${ }^{1}$ Fisioterapeuta. Mestranda em Processos Interativos dos Órgãos e Sistemas pela UFBA; ${ }^{2}$ Doutora em Medicina e \\ Saúde pela UFBA; ${ }^{3}$ Doutora em Processos Interativos dos Órgãos e Sistemas pela UFBA
}

\begin{abstract}
Resumo
Objetivo: o estudo tem por objetivo apresentar dados sobre os registros de Desordens Congênitas em menores de 1 ano no Estado da Bahia entre 2012 e 2016. Metodologia: estudo epidemiológico e descritivo, com obtenção de dados secundários do Departamento de Informática do Sistema Único de Saúde (DATASUS), no período de 2012 a 2016, por meio de consulta no SIHSUS (Sistema de Informações Hospitalares do Sistema Único de Saúde), Sistema de Informações sobre Nascidos Vivos - SINASC, e Estatísticas Vitais do TABNET disponibilizados nesta plataforma. Resultados: foi observado um total de 7406 nascidos vivos por ocorrência de anomalias congênitas neste período, onde o maior número de casos foi registrado em 2016 (23,95\%). A média de nascidos vivos com anomalias congênitas corresponde 2.468 indivíduos. As os defeitos congênitos de maior incidência se referem a malformações e deformidades congênitas do aparelho osteomuscular (48,04\%). As alterações congênitas que registraram maior morbidade estavam relacionadas àquelas originadas do aparelho circulatório ( $26,75 \%$ de todos os casos de internação), sendo também as principais causas de óbito neste mesmo período (38,07\%). Conclusão: nesta série histórica observa-se que as anomalias são as mais frequentes em nascidos vivos no período de 2012 a 2016, onde malformações congênitas do aparelho circulatório as de maior causa de morbidade e mortalidade no primeiro ano de vida. Um número de 1060 óbitos por malformações cardíacas e circulatórias em menores de 1 ano de vida foi registrado a despeito de um total de nascidos vivos de 161 indivíduos registrados nessa base de dados. Acreditamos que a divergência dessas informações possa ter ocorrido por falha na alimentação dos dados, pela forma da implantação do e pelas dificuldades quanto à interpretação e o uso do CID-10. A alimentação inadequada das informações pelo DATASUS limita a representação real dos valores para se construir um mapeamento do comportamento das malformações congênitas e suas repercussões em saúde pública. Palavras-chave: Anomalias Congênitas. Morbidade. Mortalidade.
\end{abstract}

\begin{abstract}
Objective: the study aims to present data about Congenital Disorders records in children under 1 year in the State of Bahia between 2012 and 2016. Methodology: epidemiological and descriptive study, with secondary data of the Data Processing Department of the National Health System (DATASUS), in the period from 2012 to 2016, by querying the SIHSUS (Hospital Information System of the National Health System), Live Births Information System - SINASC, and Vital statistics of TABNET available on this platform. Results: it was observed a total of 7406 live births for occurrence of congenital anomalies in this period, where the largest number of cases was registered in 2016 (23,95\%). The average number of live births with congenital anomalies corresponds to 2,468 individuals. Congenital defects of greater incidence refer to congenital deformities and malformations of the musculoskeletal system (48,04\%). Congenital changes that recorded greater morbidity were related to those originating from the circulatory system (26,75\% of all cases of hospitalization), also recorded as the main causes of death in this same period (38,07\%). Conclusion: in this historical series it is noted that the anomalies in live births are the most frequent in the period from 2012 to 2016, where congenital malformations of circulatory system are the leading cause of morbidity and mortality in the first year of life. A number of 1060 deaths caused by cardiac and circulatory malformations in children under 1 year of life was recorded despite a total live births of 161 individuals registered in this database. We believe that the divergence of this information may have been due to failure in data feed, by means of its deployment and by the difficulties concerning the interpretation and the use of CID-10. The inadequate feed of information by the DATASUS limits the actual representation of the values to construct a behavior mapping of congenital malformations and its impact on public health. Keywords: Congenital Anomalies. Morbidity. Mortality.
\end{abstract}

\section{INTRODUÇÃO}

Podem ser consideradas como Anomalias Congênitas (AC) quaisquer anormalidades estruturais ou funcionais que ocorreram em um indivíduo desde o seu nascimento, podendo estarem relacionadas a defeitos genéticos primários ou até mesmo por indução por uso de fárma-

Correspondente/Corresponding: * Maria Betânia Pereira Toralles - Instituto de Ciências da Saúde - Universidade Federal da Bahia. Av. Reitor Miguel Calmon s/n - Vale do Canela. CEP 40.110-100. Salvador, Ba - Tel.: (71)99965-9211 - E-mail: m.toralles@uol.com.br cos ou substâncias nocivas durante a gravidez, além de possíveis alterações no líquido amniótico (OMS, 2015). Esses fatores podem levar a distúrbios de desenvolvimento com um elevado índice de morbidade, e possui grande representatividade entre as causas de mortalidade infantil (MENEZES et al., 1996). Existe uma dificuldade de se ter precisão sobre a incidência de defeitos congênitos porque os casos de morte fetal antes do diagnóstico da gravidez não são muito claros (HANAOKA et al., 2018). A prevalência mundial de alterações congênitas em nascidos vivos é calculada em aproximadamente $2 \%$ a $5 \%$ (CANAKU 
et al., 2014; COSTA; GAMA; LEAL, 2006; MATTOS; GIUGLIANI; HAASE, 1987). No entanto, a grande maioria das mortes por AC ocorrem durante o primeiro ano de vida, impactando na taxa de mortalidade infantil (AMORIM et al., 2006). Tais anomalias podem ser classificadas em categorias a saber: síndromes cromossômicas, síndromes monogênicas, fatores ambientais, anomalias congênitas isoladas, e anomalias congênitas múltiplas (GARNE et al, 2011). É necessária a compreensão de que defeitos congênitos menores são os de maior incidência (OMS, 2015) e que podem ser expressados apenas com o passar dos anos. Um estudo americano aponta uma possibilidade cinco vezes maior de malformações entre os óbitos fetais, sendo as anomalias do sistema nervoso central as mais frequentes (COPPER et al., 1994), reforçando a hipótese de que defeitos congênitos de maior magnitude podem levar a resultados letais. Estudos consideram que pelo menos $5 \%$ dos nascidos vivos pode apresentar algum tipo de malformação e que fatores genéticos poderiam ser sua determinante (HOROVITZ; LLERENA JUNIOR; MATTOS, 2005). As etiologias eram desconhecidas até que avanços na medicina e nos estudos em genética pudessem trazer respostas para esses achados. As determinantes genéticas das doenças e o sequenciamento do genoma possibilitaram mensuração de agravos à saúde e ajudaram na identificação de mutações em genes que poderiam estar relacionados a muitos insultos de causas não identificadas (AUFFRAY et al., 2011). A partir desses dados, doenças mendelianas podem agora ser encontradas e mapeadas e seus mecanismos podem então ser compreendidos explorando os genes com potencialidade de causarem uma dada doença (POLIAKOV et al., 2015).

Ações não-governamentais e de livre iniciativa foram desenvolvidas com foco na atenção às anomalias congênita, a exemplo do Estudo Colaborativo Latino-Americano de Malformações Congênitas (ECLAMC), o SIAT - Sistema Nacional de Informação sobre Agentes Teratogênicos dispõe de informações sobre riscos de exposição de gestantes a agentes químicos, físicos e biológicos, sendo o ECLAMC considerado um dos mais importantes programas de monitoramento das malformações congênitas (MEIRA; ACOSTA, 2009). Existe uma dificuldade na deteç̧ão de malformações a exemplo das cardiopatias e anomalias do sistema digestivo em comparação das ocorrências no sistema genital e osteomuscular, o que pode resultar em subnotificações. Outra barreira para esses diagnósticos pode ser sobre o inadequado conhecimento dos profissionais sobre o segundo e terceiro dígito do CID-10, o que requer treinamento e capacitação para preenchimento regular da Declaração de Nascidos Vivos (DNV). (LUQUETTI; KOIFMAN, 2010; NHONCANSE; MELO, 2012).

O presente estudo propõe apresentação de dados sobre registros de desordens congênitas no Estado da Bahia, auxiliando o processo de compreensão na interpretação desses dados para que se possam nortear o estabelecimento de prioridades e planejamento e ações voltadas para este cenário.

\section{METODOLOGIA}

Trata-se de um estudo epidemiológico e descritivo, com obtenção de dados secundários do Departamento de Informática do SUS (DATASUS), no período de 2012 a 2016. Tais dados foram obtidos por meio de consulta às seguintes bases de dados: SIH SUS (Sistema de Informações Hospitalares do Sistema Único de Saúde), Sistema de Informações sobre Nascidos Vivos - SINASC e Estatísticas Vitais do TABNET, disponibilizados pelo Departamento de Informática do Sistema Único de Saúde (DATASUS), no endereço eletrônico (http://www.datasus.gov.br), que foi acessado em 21/06/2018. A população do estudo se deu através dos casos de internação hospitalar e óbitos por anomalias congênitas entre menores de 1 ano, diagnosticados e registrados no período de 2012 a 2016, além de nascimento por ocorrência de defeitos congênitos do tipo: espinha bífida, outras malformações congênitas do sistema nervoso, malformações congênitas do aparelho circulatório, fenda labial e fenda palatina, ausência, atresia e estenose do intestino delgado, outras malformações congênitas aparelho digestivo, testículo não-descido, outras malformações do aparelho geniturinário, deformidades congênitas do quadril, deformidades congênitas dos pés, outras malformações e deformidades congênitas do aparelho osteomuscular, outras malformações congênitas, anomalias cromossômicas NCOP (não classificadas em outra parte). Não usamos dados referente ao período de 2017 por não haver registro suficiente neste período. A partir das informações obtidas no DATASUS, reorganizamos, analisamos e desenhamos novas tabelas por meio do programa Excel. Em se tratando deste banco ser de domínio público, não houve necessidade de submissão ao Comitê de Ética em Pesquisa.

\section{RESULTADOS}

A análise dos dados traz no período de 2012 a 2016 um total de 7406 nascidos vivos por ocorrência de anomalias congênitas, com registro de 1424 casos em 2012 (19,22\%), 1395 em 2013 (18,83\%), 1298 em 2014 (17,52\%), 1515 em 2015 (20,45\%) e 1774 em 2016 (23,95\%), o que significa uma maior incidência registrada no ano de 2016. A média de nascidos vivos com anomalias congênitas neste período corresponde 2.468 indivíduos. As anomalias congênitas de maior incidência se referem a malformações e deformidades congênitas do aparelho osteomuscular $(48,04 \%)$, seguidas das malformações congênitas do sistema nervoso $(16,74 \%)$, malformações do aparelho geniturinário $(8,81 \% \%)$, malformações congênitas aparelho digestivo $(8,47 \%)$, anomalias cromossômicas $(3,01 \%)$ e malformações congênitas do aparelho circulatório (2,17\%). A Figura 1 representa graficamente os achados alo longo dos anos. Nota-se que a variação de nascimento por ocorrência de defeitos congênitos não sofre grandes variações na cronologia, exceto ao que concerne ao sistema nervoso, onde se observa um aumento na incidência a partir de 2014, saindo de um 
percentil de $9,01 \%$ para $27,45 \%$ em 2016 . As alterações do aparelho musculoesquelético também sofrem variações em sua ocorrência no mesmo período, porém em queda (de 50,14\% em 2014 para 42,22\% em 2016). As demais anomalias não apresentam variação significante quando comparadas a esses achados.

Figura 1-Representação gráfica em percentis dos nascidos vivos por ocorrência de malformações congênitas de 2012 a 2016.

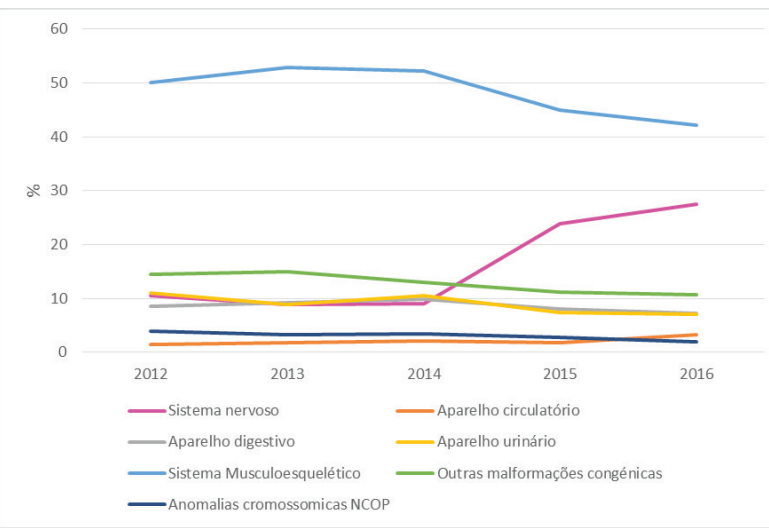

Fonte: Autoria própria

Com relação à ocorrência de morbidade relacionada a estas anomalias em menores de 1 ano de vida, observa-se que entre 2012 a 2016 houve um número de internações de 5116 indivíduos que apresentavam algum tipo dessas malformações congênitas, sendo em 851 casos em 2012 (16,63\%), 949 casos em 2013 (18,54\%), 1058 casos em 2014 (20,68\%), 1153 casos em 2015 (22,53\%) e 1105 casos em 2016 ((21,59\%), sendo em 2015, portanto, o período de mais internações por ocorrências de AC. A média de internações no período é de 1023 casos. As malformações do aparelho circulatório foram as maiores responsáveis pelas internações nesse período ( $26,75 \%$ das ocorrências), seguidas das malformações osteomusculares $(21,44 \%)$ e as do sistema digestivo (20,28\%). A Figura 2 representa graficamente esses achados ao longo dos anos.

Figura 2 - Internação por ocorrência de defeitos congênitos de 2012 a 2016.

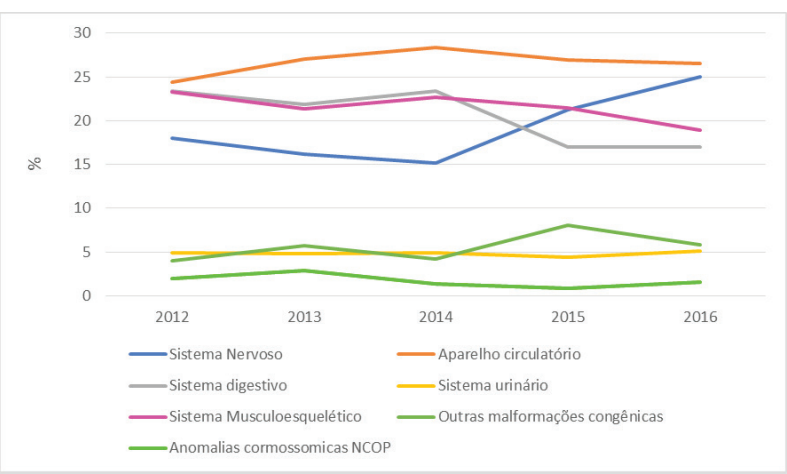

Fonte: Autoria própria
Dentre os registros de óbitos alimentados nessa base de dados a maior frequência observada foi por ocorrência de defeitos no aparelho circulatório $(38,07 \% 0$, seguida das alterações do sistema nervoso $(19,26 \%)$ e das alterações do sistema osteomuscular (9,79\%). Os demais achados podem ser vistos na Figura 3.

Figura 3 - Frequência de óbitos por ocorrência de defeitos congênitos

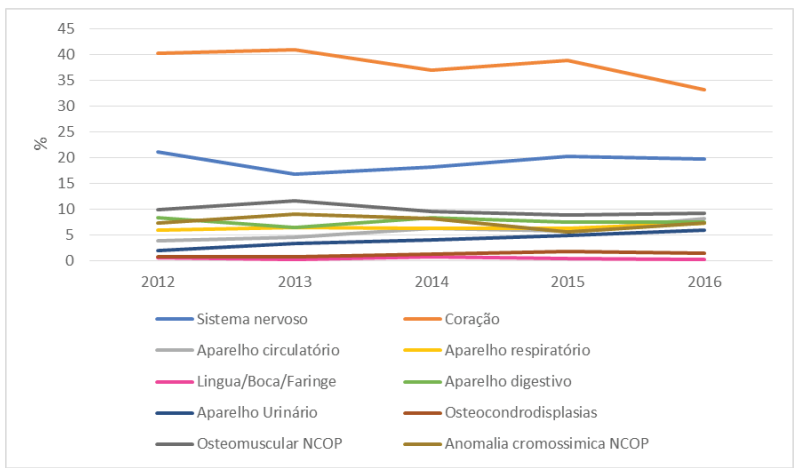

Fonte: Autoria própria

\section{DISCUSSÃO}

O Departamento de Informática do Sistema Único de Saúde existe desde 1991 e tem como competência gerir os sistemas de informação do SUS que são necessários ao processo de planejamento, operação e controle. Atualmente é responsável por apresentar elucidações de software para as secretarias estaduais e municipais de saúde de acordo com as necessidades de seus gestores. (DATASUS, 2018).

Nosso estudo traz as anomalias musculoesqueléticas como principal ocorrência de defeitos congênitos em nascidos vivos no período de 2012 a 2016 (48,04\%), sendo as malformações congênitas do aparelho circulatório as de maior causa de morbidade no mesmo período $(26,75 \%$ ) e os defeitos cardíacos com as alterações do aparelho circulatório as principais causas de óbitos até o primeiro ano de vida $(43,81 \%)$. Durante a nossa análise, observamos uma dificuldade quanto a coleta dos dados de nascimento por ocorrência de defeitos cardíacos; o campo "Cardiopatias Congênitas", contido no Sistema de Informações sobre Nascidos Vivos - SINASC não possui informações alimentadas. Tais informações foram conseguidas apenas na coleta de dados no item "malformações congênitas do aparelho circulatório". Os dados específicos sobre defeitos intracardíacos (patologias do miocárdio) e do aparelho circulatório aparecem melhor descritos no Sistema de Informações sobre Mortalidade - SIM, separados por categorias do CID-10. É possível que a inexistência desses dados sobre nascimento por ocorrência de Cardiopatia Congênita se justifique pela necessidade da realização de ecocardiograma nos recém-nascidos, não disponível em todas as maternidades e, em determinados casos, acaba por ser feito um diagnóstico em fase mais tardia (RAMOS, 2008). 
Comparando-se os 3 gráficos apresentados neste trabalho, podemos observar entre os nascidos por ocorrência de AC uma similaridade entre as categorias com exceção às classificadas como "musculoesqueléticas", que se apresentam com achados mais significativos que os demais. No gráfico de morbidades malformações no sistema urinário, anomalias cromossômicas e outras malformações congênitas são as que apresentam menor frequência de internações, quando comparada às demais AC. E, por fim, no gráfico de óbitos por ocorrência de malformações, os defeitos cardíacos e neurológicos se apresentam como os mais relevantes em termo de mortalidade, dado semelhantemente apresentado em outro estudo (RAMOS, 2008). Não encontramos nenhum estudo que justificasse a relação existente entre tempo de morbidade e mortalidade por AC nesta faixa etária.

É importante lembrar que estudos baseados em dados secundários apresentam algumas limitações, em se pensar que muitos dos campos analisados podem não estar preenchidos adequadamente, mascarando as informações, como se pôde observar neste estudo. Prova disso se dá, por exemplo, ao verificar a frequência de nascidos por ocorrência de anomalias no aparelho circulatório em comparação ao número de óbitos relacionados a este defeito; nesta série histórica observa-se um número de 1060 óbitos por malformações cardíacas e circulatórias em menores de 1 ano de vida, sendo um total de nascidos vivos de 161 indivíduos registrados nessa base de dados, uma discrepância altamente significante. Essa divergência de informações pode ter ocorrido não somente pela falha na alimentação dos dados, mas também pala maneira de como o sistema foi implantado, a organização do mesmo e o desenvolvimento desses sistemas que sofrem variações em se pensar da forma como ocorreu em cada região (COSTA et al., 2003). A ausência de diagnóstico das malformações cardíacas após o nascimento também é uma possível causa para falhas na alimentação desses dados, além das dificuldades quanto à interpretação e o uso do CID-10. O efeito disso a longo prazo leva a traduções equivocadas e dificuldade de implantação de políticas públicas voltadas para esses achados. Um estudo de 2017 reforça que a precocidade do diagnóstico do defeito cardíaco possa antecipar cuidados em saúde e melhorando a sobrevida dos recém-nascidos (LIMA et al., 2017). Essa dificuldade na alimentação das informações pelo DATASUS limita a representação real dos valores para se construir um mapeamento do comportamento das malformações congênitas e suas repercussões em saúde pública. As falhas de alimentação desses dados podem justificar as divergências também encontradas em estudos semelhantes ao nosso.

A evolução do diagnóstico genético permitiu a compreensão do surgimento de uma infinidade de doenças até então de etiologia desconhecida. $\mathrm{O}$ aperfeiçoamento dentro do campo na genética permitiu a avaliação sobre as probabilidades de uma doença acontecer, evitando assim a sua patogenia (ALBANO, 2000). Em janeiro de 2009, o Ministério da Saúde criou a Portaria GM no 81, fornecendo no escopo do Sistema Único de Saúde/SUS a Política Nacional de Atenção Integral em Genética Clínica, auxiliando na prevenção precoce das AC. Neste aspecto cria-se uma rede de serviços regionalizada e hierarquizada que atuam especificamente na Atenção Básica e famílias e indivíduos com problemas relacionados a $\mathrm{AC}$ e patogenias genéticas são identificadas e acompanhadas do ponto de vista genético (BRITO et al., 2010). A compreensão da história familiar em indivíduos que apresentam algum tipo de malformação congênita pode, através do aconseIhamento genético, antecipar os cuidados para prevenir complicações, identificar familiares em risco de evoluir com defeitos congênitos previamente desconhecidos e impactar no manejo, na decisão terapêutica e consequentemente, em ações que possam melhorar do prognóstico dos pacientes afetados.

\section{REFERÊNCIAS}

ALBANO, L. M. J. Importância da genética no serviço público: relato da extinção de um setor de genética no município de São Paulo, Brasil. Rev. panam. salud pública, Washington, v.7, p. 29-34, 2000.

AMORIM, M. M. R. D. et al. Impacto das malformações congênitas na mortalidade perinatal e neonatal em uma maternidade-escola do Recife. Rev. bras. saúde matern. Infant., Recife, s19-s25, 2006.

AUFFRAY, C. et al. Genome Medicine: past, present and future. Genome med.,[S.I], v.3, n. 6, 2011.

BRITO, V. R. S. et al. Anomalias congênitas e fatores de risco materno em Campina Grande-Paraíba. Rev. Rene, Fortaleza, v. 11, n. 2, p. 10, 2010.

CANAKU, D. et al. Prevalence and factors associated with congenital malformations in tirana, Albania, during 2011-2013. Mater. Sociomed., Sarajevo, v. 26, n. 3, p. 158-612, June 2014.

COPPER, R. L. et al. Risk Factors for fetal death in white, black and Hispanic women. Colaborative Group on Preterm Birth Prevention. Obstet gynecol., Hagerstown, v. 85, p. 318-319, 1994.

COSTA, C. M. da. S.; GAMA, S. G. N. da.; LEAL, M. do. C. Congenital malformations in Rio de Janeiro, Brazil: prevalence and associated factors. Cad. saúde publica, Rio de Janeiro, v. 22, n. 11, p. 2423-2431, Nov.2006.

COSTA, M. da C. N. et al. Mortalidade infantil no Brasil em períodos recentes de crise econômica. Rev. Saúde Pública, São Paulo, v.37, n.6, p. 699-706, 2003.

DATASUS. 2018. Disponível em:<http://datasus.saude.gov.br/datasus>. Acesso em: 04 ago. 2018.

GARNE, E. et al. Paper 5: surveillance of multiple congenital anomalies: implementation of a computer algorithm in european registers for classification of cases. Birth Defects Res. Part. A Clin. Mol. Teratol., Hoboken, v. 91, n. S1, p. S44-S50, 2011.

HANAOKA, T. et al. Prevalence and risk of birth defects observed in a prospective cohort study: the hokkaido study on environment and children's health. J. epidemiol., Tokyo, v. 28, n.3, p.125-132, 2018.

HOROVITZ, D. D. G.; LLERENA JUNIOR, J. C.; MATTOS, R. A. de. Atenção aos defeitos congênitos no Brasil: panorama atual. Cad. saúde pública, Rio de Janeiro, v.21, n. 4, p.1055-1064, jul./ago. 2005.

LIMA, I. D. de et al. Perfil dos óbitos por anomalias congênitas no Estado do Rio Grande do Norte no período de 2006 a 2013. Rev. Ciênc. Méd. Biol., Salvador, v. 16, n. 1, p. 52-58, 2017. 
LUQUETTI, D. V.; KOIFMAN, R. J. Qualidade da notificação de anomalias congênitas pelo Sistema de Informações sobre Nascidos Vivos (SINASC): estudo comparativo nos anos 2004 e 2007. Cad. saúde pública, Rio de Janeiro, v. 26, n. 9, p.1756-1765, 2010.

MATTOS, T. C.; GIUGLIANI, R.; HAASE, H. B. Congenital malformations detected in 731 autopsies of children aged 0 to 14 years. Teratology, Philadelphia, v. 35, n. 3, p. 305-357, June 1987.

MEIRA, J. G. C.; ACOSTA, A. X. Políticas de saúde pública aplicadas à genética médica no Brasil. R. Ci. méd. biol., Salvador, v.8, n.2, p. 189197, mai./ago. 2009.

MENEZES, A. et al. Mortalidade infantil em duas coortes de base populacional no Sul do Brasil: tendências e diferenciais. Cad. saúde pública, Rio de Janeiro, v.12, p. S79-S86, 1996.
NHONCANSE, G. C.; MELO, D. G. Confiabilidade da declaração de nascido vivo como fonte de informação sobre os defeitos congênitos no Município de São Carlos, São Paulo, Brasil. Ciênc. Saúde Coletiva, Rio de Janeiro, v.17, n. 4, p. 955-963, 2012.

ORGANIZAÇÃO MUNDIAL DE SAÚDE(OMS)/CDC/ICBDSR. Vigilancia de anomalías congénitas: manual para gestores de programas. Ginebra: Organización Mundial de la Salud, 2015.

POLIAKOV, E. et al. Genetics in genomic era. Genet. res. int., London, v. 2015, 2015.

RAMOS, A. P. Prevalência de malformações congênitas em recém-nascidos em hospital da rede pública. Revista saúde. com., Jequié, v. 4, n.1, p. 27-42, 2008.

Submetido em: $14 / 11 / 2018$

Aceito em: 29/11/2018 